\title{
Development of Automatic Detection System of Miniature Voltage Electromagnetic Relay
}

\author{
Chen Shaoke \\ Mechatronic Engineering Department \\ Shantou University \\ Guangdong, China \\ e-mail:skchen@stu.edu.cn \\ Peng Jiangbo \\ Mechatronic Engineering Department \\ Shantou University \\ Guangdong, China \\ e-mail: fbpeng@stu.edu.cn
}

\author{
Xu Qihang \\ Department of Machinery\& Electric \\ Jieyang Vocational and Technical College \\ Guangdong, China \\ e-mail: xuqihang306@126.com
}

\begin{abstract}
With the Electromagnetic relay development, the detection technology of relay is more advanced. At the same time, users are also increasingly high requirements on... quality, performance and reliability of relay. This requires very precise relay detection, in order to ensure the quality and reliability of relay production. The combination of the analog and digital electronic technology and computer technology, completing automatic detection of the relevant parameters on voltage electromagnetic relay is the development direction of electromagnetic relay detection. This paper mainly introduces the main function and work principle of voltage electromagnetic relay detection system based on industrial computer as the core. The miniature relay parameter test instrument is developed and the rate of qualified products is count. The product quality is improved, and it has the advantages of convenient operation and high accuracy rate. The system is suitable for small and mediumsized enterprises voltage electromagnetic relay factory test, it provides a powerful reference for the development of automatic detection system.
\end{abstract}

Keywords-Relay; Electromagnetic; Industrial Personal Computer; Automatic Measuring; Excitation Current

\section{INTRODUCTION}

Electromagnetic relay has the high insulation resistance as its off state and Low conduction resistance as its on state thus other electronic components cannot be compared with it. It is one of the most widely used electrical power and other electrical systems. In all fields of national economy, especially it occupies an important position in the national key military engineering. It is used widely in automatic control system, remote control, electric power system, protection system and communication system. It plays the role of control, detection, protection and regulation; it is one of the most basic parts of modern electrical drive [1]. Because its manufacturing process manual procedures is more with using range of relay expand, corresponding to the various aspects performance of relay is also increasing, so the relay performance test is also very important. Manufacturing test level of electromagnetic relay has already development from manual to automatic test [2]

At present, the automation detection level of relay by the relay-contact control is getting to the PLC control and further development to the computer control. The acquisition and processing technology of the relay electrical parameter is an important sign of advanced equipment in the relay detection. In the general relay testing equipment, the transient waveform of the main current, contact voltage and power voltage are generally measured. The traditional detection is mainly light oscilloscope that is oscilloscope with light sensitive paper light records. Because the record time is long for the this structure, the number of electrical isolation channel for each is more (up to 16), the oscillator adopts suspension structure, mechanical inert ia is big, So the detection signal frequency cannot be too high as the equipment caused by detection speed is limited. The power factor, time constant, power frequency etc. cannot be measured [3-4].

This paper presents development of complete parameter test instrument of small relay. Test content includes the contact resistance, voltage, release voltage, excitation current, action time, release time, contact transfer time, noise and light test. Meanwhile it makes statistics on the rate of qualified products, and improves product quality and convenient operation. The device is composed of an industrial control computer and some peripheral circuit equipment, represents the level of automation in low voltage electrical apparatus test.

\section{THE SYSTEM DESIGN AND FUNCTION}

This testing system is designed for the detection of the relay contact and relay coil portion. The overall system principle detection diagram is shown in Fig .1. The whole test system is divided into the coil loop and contact circuit. Two circuits is isolated full electrical and has logic relation through the computer and its peripheral equipment. 
The coil circuit is used to relay the exciting current testing and provided excitation conditions for test relevant parameters of contact circuit. The contact circuit is used to test the contact resistance and the relay time parameter [5].

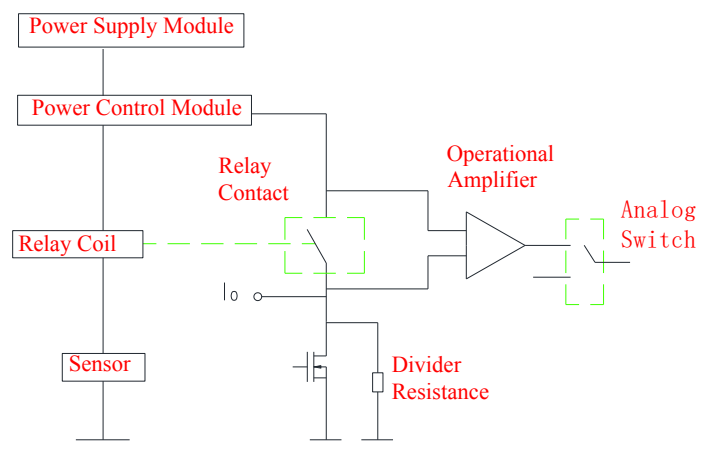

Figure 1. Overall system principle detection diagram

The system hardware block diagram shown in Fig .2. The system is mainly composed of industrial computer (IPC), analog function components, logic function component and power supply. The IPC is mainly to test data are calculated and the logic control of the test system. The powerful computing ability ensures to the multi parameter test can be Complete in the short time. The test circuit is switched by relay array. So the same hardware equipment can realize different logic functions. The system is controlled test circuit transformation by IPC controlled relay array transformation. At the same time it is isolate between the IPC and the control circuit. The power part provided the load power for relay contacts. The relay coil provides encouraging conditions and the working power for some devices in the test equipment [5].

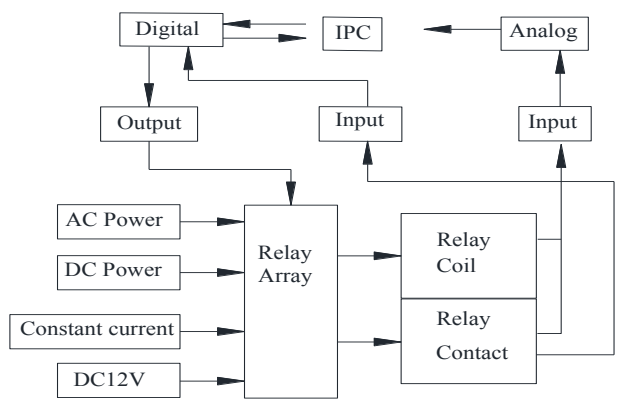

Figure 2. System hardware structure block diagram

The main function of the test bench:

- Complete tests of the relay voltage, voltage release, action time, release time, transfer time, excitation current, contact resistance, light emitting component on the same station.

- Real time displays the current detection parameters and failure parameters of each relay. Check out the relay to determine whether qualified, and statistics number of test qualified and unqualified.

- With man-machine conversation function, manually set the relay parameters. Effectively meet the non-standard relay testing.
- The test bench has the advantages of convenient operation, visual display.

\section{THE HARDWARE CIRCUIT DESIGN}

\section{A. Test circuit of relay excitation current}

Measuring relays magnetizing current is loading rated voltage in both sides of relay coil. At the same time produce excitation current in the measurement coil circuit. The test circuit diagram is shown in Fig .3. In the system, the current of coil circuit is converted linear into 0 $\sim 3.5 \mathrm{~V}$ voltage signal through $\mathrm{WB}$ AC and DC electrical isolation sensor. This signal is input computer by the $\mathrm{A} / \mathrm{D}$ acquisition card. The exciting current value of relay coil is calculate by the software and displayed on the screen. Because the relay model is various with $\mathrm{AC}$ and $\mathrm{DC}$ relay so exciting current range is wide. In order to ensure the accuracy of measurement, electrical isolation sensor is used two range $0 \sim 50 \mathrm{~mA}$ and $0 \sim 200 \mathrm{~mA}$. When the excitation current is less than $50 \mathrm{~mA}$ selects $0 \sim 50 \mathrm{~mA}$ power sensor by industrial control computer magnetic latching relay. When the excitation current is greater than $50 \mathrm{~mA}$ selects 0 $200 \mathrm{~mA}$ power sensor by the magnetic latching relay.

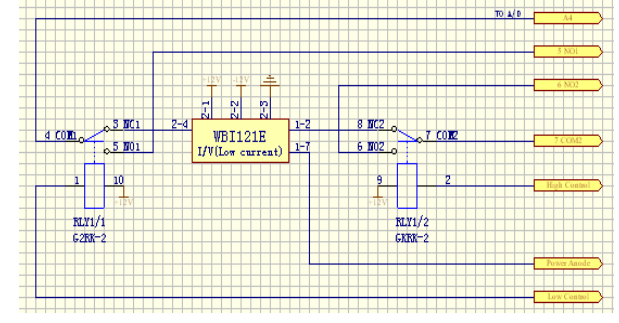

Figure 3. Excitation current test circuit diagram

\section{B. Contact state detection circuit}

When the relay is inserted into the socket for detect, must first detect contact of each contact of relay. Relay contacts each contact each other are insulated. They were driving an armature, coordinated action. When the armatures suck up, each movable contact simultaneously open with the post contact and connected with the front contact. When the armatures fall down, each movable contact simultaneously open with the front contact and connected with the post contact. Contact state detection circuit diagram is shown in Fig .4. In this system the whole system is reset first by the IPC. When the start test, $12 \mathrm{~V}, 3 \mathrm{~mA}$ current is load in the contact circuit. The contact voltage will compared with the calculated reference voltage (threshold) through integrated block of a LM339 voltage comparator. LM339 "+" is threshold level, "-" is connected with a detected relay contact. Both ends of the voltage signal is detected. When the "+" terminal voltage higher than the "" end, the output pipe cut-off. Show the pressure drop is too large so that contact not effectively closed. When the "" end voltage higher than the "+" end, the output transistor saturation. Equivalent to the output end connected to a low potential. Show contact effective contact. The two input voltage difference is greater than $10 \mathrm{mV}$ can ensure the output can be reliably into another state from one state. Therefore, the use of a voltage comparator LM339 can effectively guarantee the accuracy 
of measurement. Considering that the switch inputs in the "on" and "off" transient very strongly interfere on computer and the strong electric field interference. After the contact signal by the voltage comparator is inputted A/D acquisition card through TLP521-4 opts isolated, the contact signal is collected into the industrial control computer.

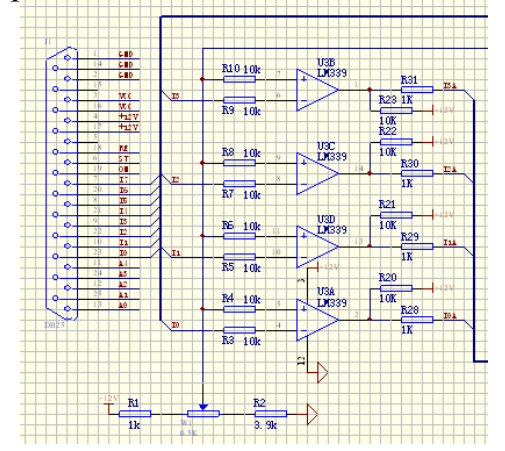

Figure 4. Contact state detection circuit diagram

\section{The contact resistance testing circuit}

The contact resistance between the contact of the relay is very small, should be less than or equal to $50 \mathrm{~m} \Omega$ in accordance with the design standards. So the pressure drop is very small at both ends of the relay contacts. Measurement of contact resistance is used a constant current source method. The relevant standard in accordance with the constant current source is used $6 \mathrm{~V}, 1 \mathrm{~A}$. Using constant current source module provides a steady current for contact. The contact resistance is measured through detecting the voltage at both ends of the relay contacts and calculating by software. The test circuit diagram is shown in Fig .5.

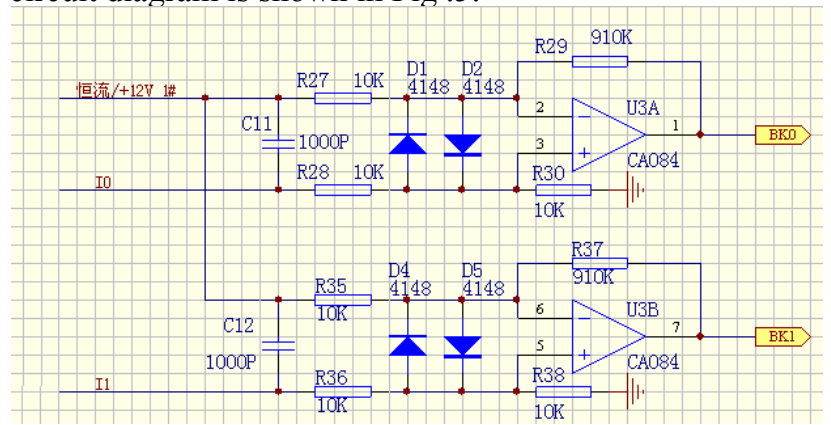

Figure 5. Contact resistance test circuit diagram

Through testing relay contacts the pressure drop at both ends of each pair of contacts and then the operational amplifier amplifies. Fig .only give the test circuit of single contact. The test system can test four pairs contact relay. By analog switch controlled in the testing process, to 8 test circuits is collected and selected. Operational amplifier selects CA084. The voltage drop value is enlarged to $0 \sim 10 \mathrm{~V}$ voltage range that $\mathrm{A} / \mathrm{D}$ data acquisition card can collect. A/D data acquisition card converts analog signals into digital signals, and calculated the contact resistance of relay contacts by IPC acquisition [6].

\section{SOFTWARE DESIGN}

Software design of the test equipment mainly includes: three modules of the user interface design, testing and monitoring functional design, parameter input module design. The program of the user interface is loaded into memory in software system initialization and provides the operating environment of software. The program of testing and monitoring is in charge of motion control of test samples, equipment line switching, sample contact signal monitoring, test failure judgment and recording failure data during the whole experiment. The main function of parameter input module program is inputting test operation parameters by the test person before the beginning of the test run. And add up number of qualified in the process of test. Allow users to test data on the hard disk copy to the floppy disk after the completion of the test. The system software block diagram is shown in Fig .6.

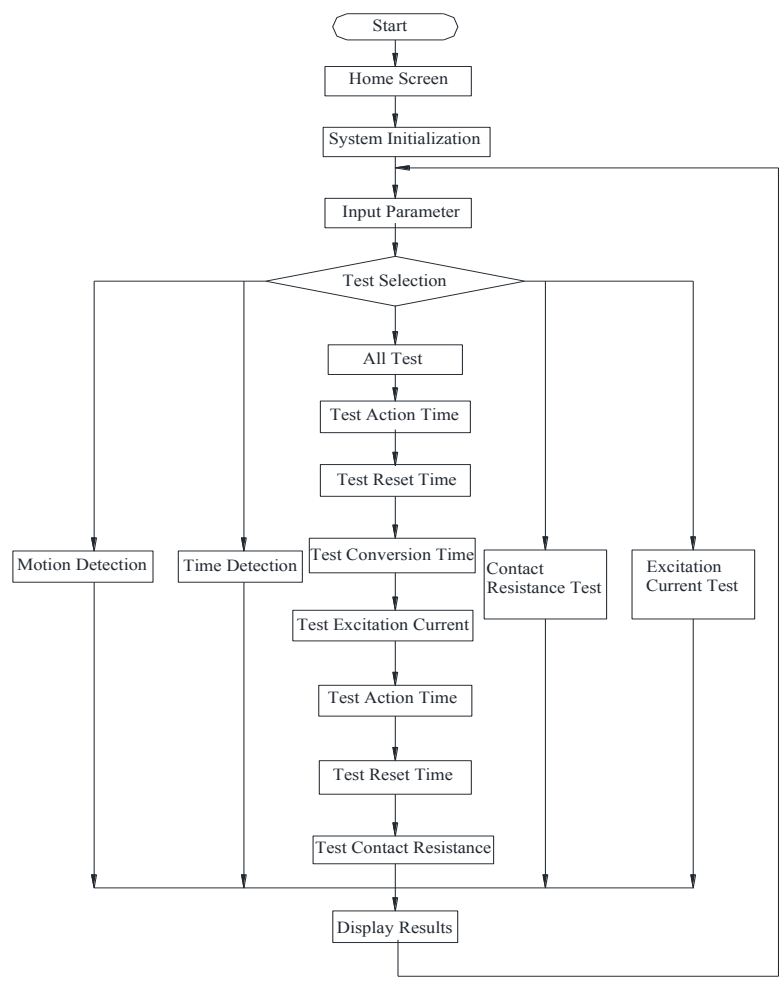

Figure 6. Block diagram of system software

The system software was programmed by $\mathrm{C}$ language. As the system on power and initialization, the corresponding relay parameters is inputted by the keyboard, and converted into a test logic circuit through the relay array. Press the start button to start the test. Screen realtime display the current detection qualified or unqualified. After testing, system through the buzzer told the qualified and unqualified. The system automatically statistics the number of qualified and unqualified.

\section{A. Contact resistance test module design}

Because the contact resistance is generally less than $50 \mathrm{~m} \Omega$. So the system resistance influence on measurement must be excluded. The measure is corrected in designing this equipment. Check the equipment through standard relay before detection. 
Software calculation eliminates the effects of measuring the system resistance.

\section{B. Design of action detection module}

The action performance testing includes relay action voltage test and reset voltage test. This system adopts step method confirmatory test. That is only testing action voltage and the reset voltage Whether or not qualified without testing the specific value of action voltage and the reset voltage [9].

\section{Design of time detector module}

The time detection module test the specific value of the relay action time, the reset time, the actions conversion time and the reset conversion time. Test block diagram is shown in Fig .7. After system test autonomic all time parameter compare with the parameter input values. The qualified time parameters display with white font and the unqualified time parameters display with red font.
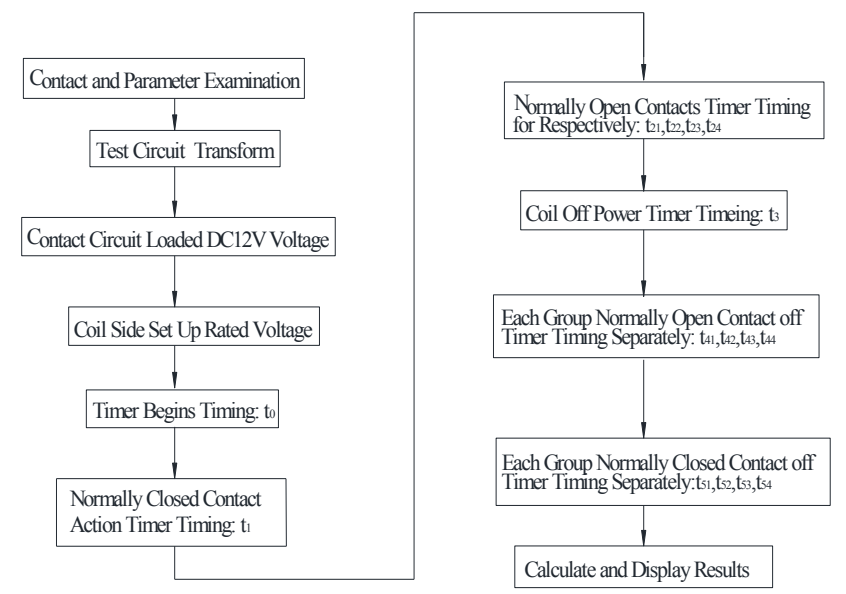

Figure 7. Time detection module diagram

\section{Design of excitation current detection module}

The excitation current testing is loading rated voltage in coil circuit. Then test current in coil circuit. Test block diagram is shown in Fig .8. Measurement of excitation current is converted by the $\mathrm{I} / \mathrm{V}$ and the $\mathrm{A} / \mathrm{D}$ acquisition card collecting voltage. Then calculate the magnetizing current value.

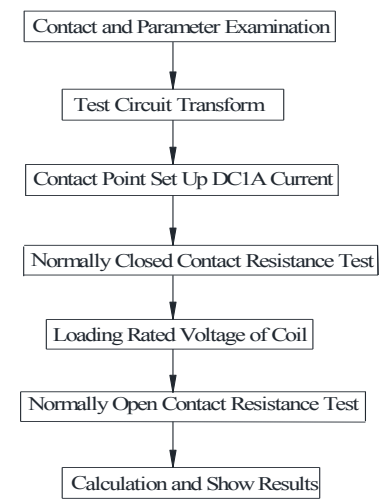

Figure 8. The excitation current detection module diagram

\section{EXPERIMENT}

The overall block diagram of test software is shown in Fig.9.

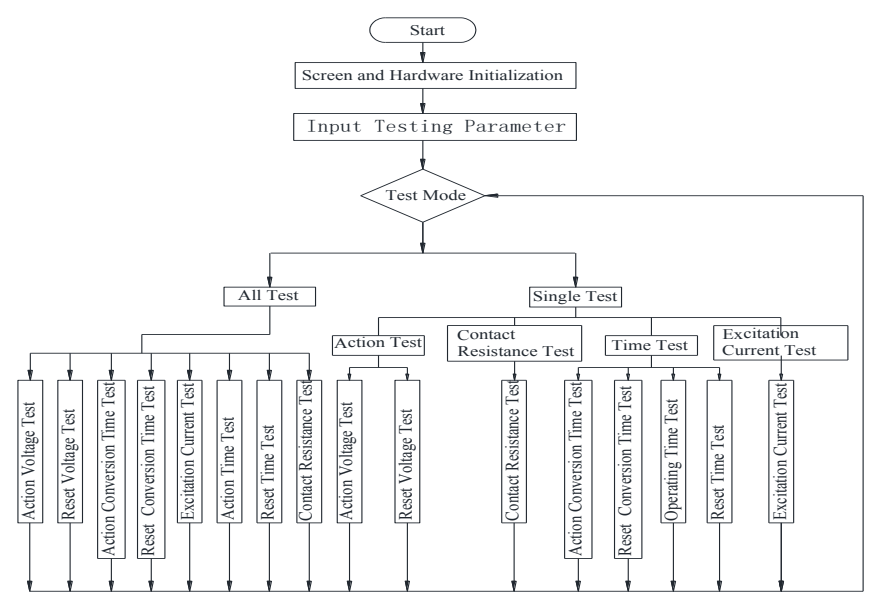

Fig .9. The overall block diagram of test software

Each test has to set up or modify the parameters at the start of the system:

- Type the detection: This system may carry on the comprehensive test for a single product. That is carrying on the continuous uninterrupted test for the electrical parameters on all the requirements of the product. Also can be single test for a single product. That is only the one phase electrical parameter of the product test. In the detection mode drop-down menu, select project of all the detection, the motion detection, the time detection, the contact resistance detection, the excitation current detection etc. One of them can be selected.

- The basic models to test products: The basic models of detection products have DY2, DY3 and DY4. The different of basic models determines the number of different relay contacts.

- Rated voltage type and rated voltage value of tested products: the AC or DC must be selected before detection rated voltage. Rated voltage value is adjusted on the external transformer by manual way. AC adjustable range is $0 \sim 280 \mathrm{~V}$ and DC adjustable range is $0 \sim 36 \mathrm{~V}$.

- Set up action voltage value of detected products: Select the action voltage settings from the menu bar. Then DC or AC power supply is adjusted to set numerical requirements by the manual adjustment. The action voltage is $78 \%$ of rated voltage value.

- Set up reset voltage value of detected products: Select the reset voltage settings from the menubar. Then DC or AC power supply is adjusted to set numerical requirements by the manual adjustment. The reset voltage is $17 \%$ of rated voltage value.

- Set up excitation current: Select the excitation current setting bar. Then input excitation current value by the keyboard.

- Set up action time: Select the action time setting bar. Then input action time value by the keyboard. 
- $\quad$ Set up reset time: Select the reset time setting bar. Then input reset time value by the keyboard.

- Set up conversion time: Select the conversion time setting bar. Then input conversion time value by the keyboard.

- Set up contact resistance: Select the contact resistance setting bar. Then input contact resistance value by the keyboard.

- $\quad$ Set up contact current: Select the contact current setting bar. Then input contact current value by the keyboard.

The program automatically save parameters the last input values. Adjust automatically parameter for the values of last set.

The main screen is the initialization window after switch on. That it is always the main window of static display during the test. On this basis, it can display the drop-down menu and pop-up windows and other information. It can be divided into four areas: Title display area, main menu area, work area, additional District title. As shown in Fig .10.

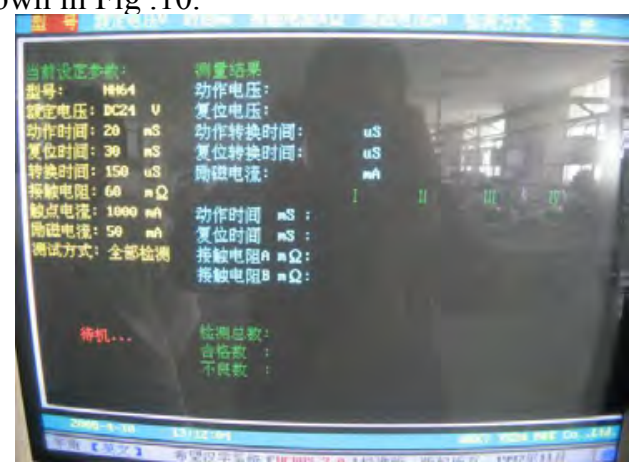

Figure 10. The main screen display window

The controlling coil side load voltage waveform by software is shown in Fig .11 when select all tests. This waveform is the loading wave of detecting DC24V. The first 5 pulse wave is used to test the relay action voltage and the reset voltage. Sixth to eighth pulse test relay time characteristic. Ninth pulse test relay contact resistance and excitation current

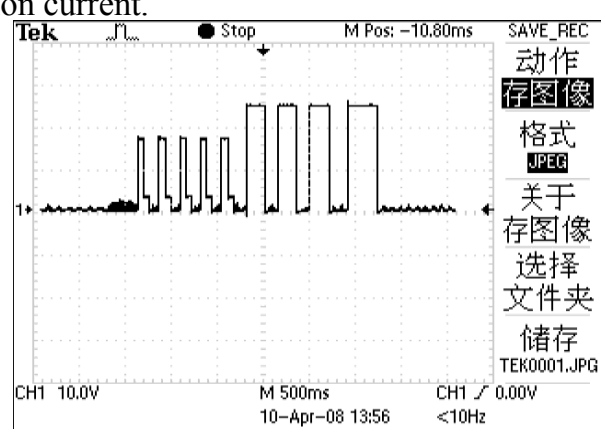

Figure 11. The software waveform of control coil side load voltage by select all testing
The device has been putted into use in three months in the factory. The system runs well. After repeated verification, its accuracy and reliability successfully passed. The device makes the factory to improve greatly detection efficiency and precision.

\section{CONCLUSION}

The equipment is combined with various means and technology at home and abroad relay testing. The relay tester of comprehensive parameters is developed in accordance with the actual needs of the product. The system can meet the accurate measurement of the operation parameters of small voltage electromagnetic relay, time parameter and contact resistance. Due to the adoption of the computer automatic control, greatly improves the efficiency and quality of production. At present the system used in the plant is good.

\section{REFERENCES}

[1] HU Zhenmin, LIU Xiaowe, JIANG Xiangang, ZHANG Hongbin and LI Guangli, "Design and Research of Relay Test System Based on PC," Journal of East China Jiaotong University, vol. 20, May. 2003, pp. 70-73

[2] WU Jincai, LIU Chengbo and WU Yongjun, "Research of an Continuous - testing Technology of Electromagnetic Relay's Electrical Performance Parameters," Electromechanical Components, vol. 32, Mar. 2012, pp. 45-52.

[3] LU Jianguo and GUO Hui, "Development of Measurement and Test Technology for Low Voltage Electrical Apparatus," Low Voltage Apparatus, vol. 1, Jan. 2002, pp. 48-52.

[4] JIANG Jingjing, "Research of Automation Test Instrument for Relay Parameter," D: Dalian. Dalian University of Technology. 2013.

[5] J. Papou`skov'a, S. Dos Santos and V. Kus, "Hysteresis of electromagnetic relays for PM Space Density Identification" CA: 13th Biennial Baltic Electronics Conference, Dec. 2012, pp. 323 326.

[6] Lee Jietae Sung, Su Whan Edgar and Thomas F, "Area methods for relay feedback tests," Industrial and Engineering Chemistry Research, vol. 49(17), 2010, pp. 7807-7813.

[7] GuofuZhai, WeiweiFan and WeiyanLiang, "Study on Contact Resistance Dynamic Characteristics of Space Electromagnetic Relay," Computational Engineering in Systems Applications, vol. 10, Oct. 2006, pp. 1074-1081

[8] Software interface design principles. http://netschool.lzu.edu.cn.

[9] HU Hongbin and FU Bin, "Testing the release value of electromagnetic relay," Electromechanical Components, vol. 2 Feb. 2004, pp. 48-52.

[10] Li Xingwen, Chen Degui and Li Zhipeng, "Numerical analysis and experimental investigation of dynamic of AC contactors concerning with the bounce of contact," CA: IEICE Transactions on Electronic, Aug. 2004, pp. 1318-1323. 